\title{
Surface-enhanced Raman Spectroscopy and Nanogeometry: the Plasmonic Origin of SERS
}

Seung Joon Lee, ${ }^{1}$ Zhiqiang Guan, ${ }^{2}$ Hongxing $\mathrm{Xu}^{2} \&$ Martin Moskovits* ${ }^{1}$

${ }^{1}$ Department of Chemistry \& Biochemistry, University of California, Santa Barbara, California 93106

${ }^{2}$ State Key Laboratory for Surface Physics, Institute of Physics, Chinese Academy of Science, China

*To whom correspondence should be addressed: mmoskovits@1tsc.ucsb.edu
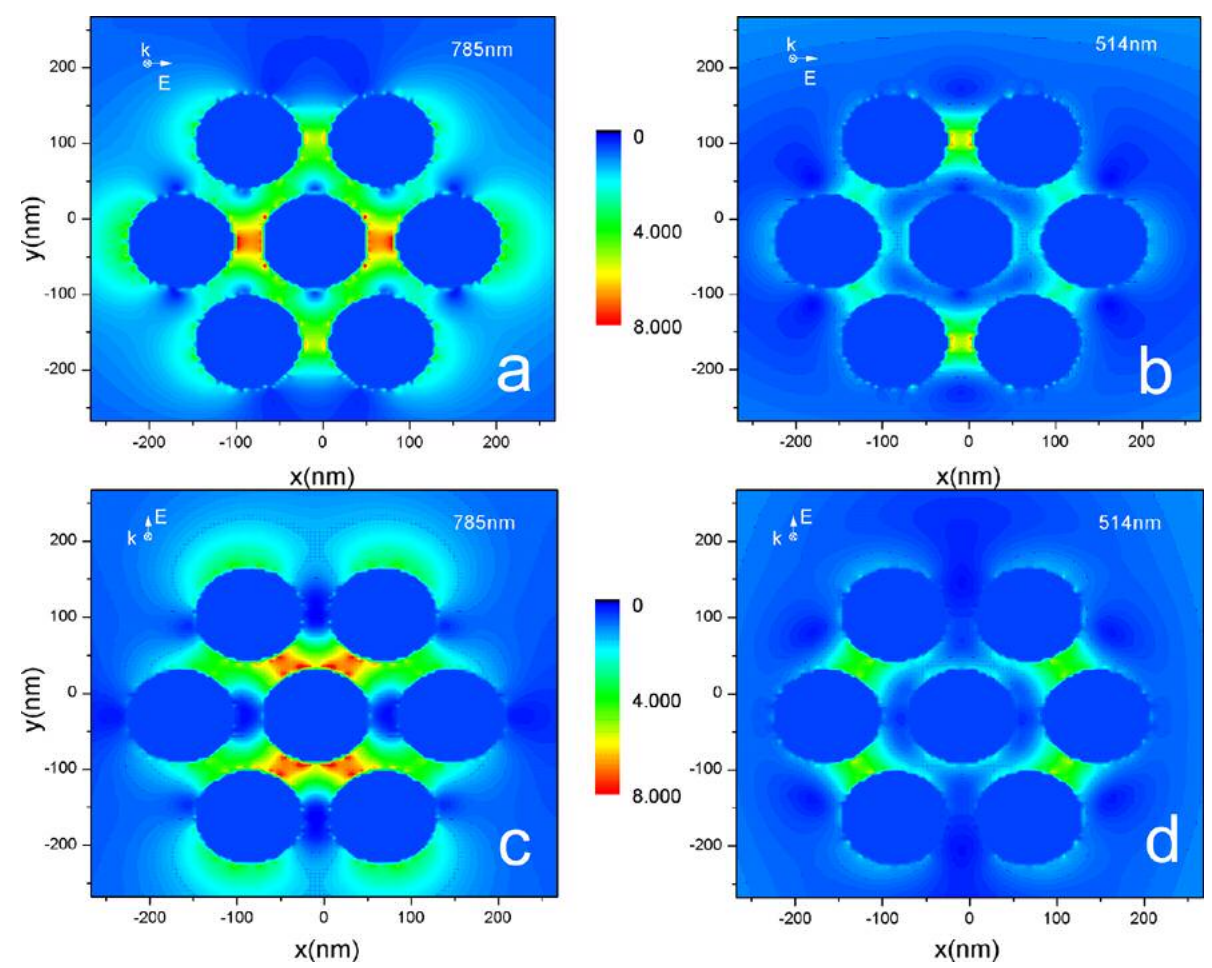

Figure S1. EM field intensity distributions calculated for a hexagonal array of seven Ag cylinders, with dimensions $80 \mathrm{~nm}$ diameter and $50 \mathrm{~nm}$ in height, and center-to-center distances of $100 \mathrm{~nm}$ corresponding to $d_{\text {gap }}=20 \mathrm{~nm}$. The polarization is shown in the figures; a and c assume $785 \mathrm{~nm}, \mathrm{~b}$ and d 514.5 excitations. 